\title{
Shikonin inhibits the Warburg effect, cell proliferation, invasion and migration by downregulating PFKFB2 expression in lung cancer
}

\author{
LIYING SHA ${ }^{1}$, ZHIQIANG LV ${ }^{1}$, YUJUN LIU ${ }^{1}$, YUN ZHANG $^{2}$, XIN SUI $^{1}$, TENG WANG $^{1}$ and HUI ZHANG ${ }^{1}$ \\ Departments of ${ }^{1}$ Pharmacy and ${ }^{2}$ Business, The Affiliated Hospital of Qingdao \\ University, Qingdao, Shandong 266003, P.R. China
}

Received June 24, 2020; Accepted February 4, 2021

DOI: $10.3892 / \mathrm{mmr} .2021 .12199$

\begin{abstract}
Lung cancer is one of the most lethal diseases and therefore poses a significant threat to human health. The Warburg effect, which is the observation that cancer cells predominately produce energy through glycolysis, even under aerobic conditions, is a hallmark of cancer. 6-phosphofructo-2-kinase/fructose-2,6-biphosphatase 2 (PFKFB) is an important regulator of glycolysis. Shikonin is a Traditional Chinese herbal medicine, which has been reported to exert antitumor effects. The present study aimed to investigate the anticancer activity of shikonin in lung cancer. Cell Counting Kit-8 (CCK-8) and colony formation assays were used to analyze proliferation in A549 and H446 cells. Wound healing and Transwell assays were used to measure migration and invasion in A549 and H446 cells. Cell apoptosis was analyzed using flow cytometry. Lactate levels, glucose uptake and cellular ATP levels were measured using their corresponding commercial kits. Western blotting was performed to analyze the protein expression levels of key enzymes involved in aerobic glucose metabolism. Reverse transcription-quantitative PCR was used to analyze the mRNA expression levels of PFKFB2. The results of the present study revealed that PFKFB2 expression levels were significantly upregulated in NSCLC tissues. Shikonin treatment decreased the proliferation, migration, invasion, glucose uptake, lactate levels, ATP levels and PFKFB2 expression levels and increased apoptosis in lung cancer cells in a dose-dependent manner. The overexpression of PFKFB2 increased the proliferation, migration, glucose uptake, lactate levels and ATP levels in lung cancer cells, while the knockdown of PFKFB2 expression exerted the opposite effects. Moreover, there were no significant
\end{abstract}

Correspondence to: Dr Hui Zhang, Department of Pharmacy, The Affiliated Hospital of Qingdao University, 16 Jiangsu Road, Shinan, Qingdao, Shandong 266003, P.R. China

E-mail: zhanghui6678@aliyun.com

Key words: shikonin, Warburg effect, 6-phosphofructo-2kinase/fructose-2,6-biphosphatase 2, migration, lung cancer differences in lung cancer cell migration, apoptosis, glucose uptake, lactate levels and ATP levels between cells with knocked down PFKFB2 expression or treated with shikonin and the knockdown of PFKFB2 in cells treated with shikonin. In conclusion, the results of the present study revealed that shikonin inhibited the Warburg effect and exerted antitumor activity in lung cancer cells, which was associated with the downregulation of PFKFB2 expression.

\section{Introduction}

Lung cancer is one of the most common causes of cancer-related mortality, accounting for $18.4 \%$ of cancer-related deaths worldwide (1). In total, $85 \%$ of lung cancer cases are diagnosed as non-small cell lung cancer (NSCLC) and $\sim 15 \%$ of lung cancer cases are diagnosed as small cell lung cancer (2). At present, the most common treatment methods for lung cancer include surgical resection, chemotherapy, radiotherapy, immunotherapy and targeted drugs (3). Although targeted therapies, such as EGFR inhibitors, and immunotherapy drugs, such as anti-programmed cell death protein 1/programmed death-ligand 1 , have significantly improved the treatment of lung cancer, only a small number of patients are eligible to be treated with targeted therapies, thus the global 5-year survival rate of lung cancer remains at $\sim 20 \%$ (4-8). Therefore, identifying specific and sensitive prognostic markers, therapeutic targets and devising novel therapeutic drugs to improve the survival time and quality of life of patients with lung cancer remains a priority.

The Warburg effect is defined by the altered metabolism of glucose, whereby tumor cells produce large amounts of lactate, even in the presence of oxygen and fully functioning mitochondria (9). The intrinsic mechanism of the Warburg effect is complex, and is associated with the activation of numerous oncogenes, inactivation of numerous tumor suppressor genes, the abnormal expression of glycosylase and changes in the tumor microenvironment, amongst other factors (10-12). However, the specific mechanism requires further investigation. It is well established that the cell death program (apoptosis) exerts an anticancer effect (13). The Warburg effect is closely associated with the occurrence and development of cancer, as it provides a growth advantage to tumor cells, helping them to 
escape apoptosis and create a suitable environment for tumor metastasis to occur (14-16). The inhibition of the Warburg effect promotes cell apoptosis and inhibits tumor proliferation and metastasis (17). Several key metabolic enzymes [pyruvate kinase M2 (PKM2), pyruvate dehydrogenase kinase 1 (PDK1) and hexokinase 2] have been found to play an important role in the Warburg effect (18-20). The changes in key enzymes can lead to an enhanced glycolytic ability, promote glucose uptake into tumor cells and increase the accumulation of lactic acid, thus further supporting tumor growth and development (21). 6-Phosphofructo-2-kinase/fructose-2,6-biphosphatase (PFKFB2) is an enzyme that regulates the synthesis and degradation of fructose-2,6-bisphosphate (Fru-2, 6-P2), which is widely expressed in a variety of cancer cells, such as ovarian, breast and pancreatic cancer cells $(22,23)$. By regulating the expression of Fru-2, 6-P2 (a signaling metabolite that participates in glycolysis), the PFKFB family has been found to regulate intracellular glycolysis in cancer by acting as an oncogene $(24,25)$.

Shikonin is a naphthalene quinone compound extracted from Lithospermum erythrorhizon (an herbaceous plant), which has demonstrated anti-inflammatory, antiviral, antitumor and wound healing potential, amongst other biological activities (26). The present study aimed to investigate the effects of shikonin on cell proliferation, migration, invasion, apoptosis and aerobic glycolysis in lung cancer cells, and further determined the potential underlying molecular mechanisms of shikonin. The current findings may provide a foundation for the clinical use of shikonin in the treatment of lung cancer.

\section{Materials and methods}

Patient studies. A total of 20 NSCLC and adjacent normal tissues were obtained from patients (10 women and 10 men; age range, 50-79 years) who underwent surgical resection at The Affiliated Hospital of Qingdao University (Qingdao, China) between January 2019 and December 2019. Patients who had received chemotherapy or radiotherapy prior to surgery were excluded from the study. Patients who were diagnosed by positron emission tomography/CT scan and were eligible for radical surgery were included in the study. All samples were stored at $-80^{\circ} \mathrm{C}$ until required for RNA extraction. The study protocols were approved by the Ethics Committee of The Affiliated Hospital of Qingdao University, and written informed consent was obtained from all patients prior to participation.

Cell lines and culture. The Beas-2B lung epithelial cell line and lung cancer cell lines, A549 and H446, were obtained from The Cell Bank of Type Culture Collection of The Chinese Academy of Sciences. All cells were cultured at $37^{\circ} \mathrm{C}$ $\left(5 \% \mathrm{CO}_{2}\right)$ in DMEM (Gibco; Thermo Fisher Scientific, Inc.) supplemented with 10\% FBS (Gibco; Thermo Fisher Scientific, Inc.) and $1 \%$ penicillin/streptomycin (Gibco; Thermo Fisher Scientific, Inc.).

Cell transfection. Small interfering RNA (siRNA)-negative control (NC) (sense, 5'-UUCUCCGAACGUGUCACG UTT-3' and antisense, 5'-ACGUGACACGUUCGGAGA
ATT-3'), siRNA-PFKFB2 (sense, 5'-AUUGUCAUGCCGAAA GAAGUC-3' and antisense, 5'-CUUCUUUCGGCAUGA CAAUGA-3'), pcDNA3.1-PFKFB2 and pcDNA3.1-NC (all Shanghai GenePharma Co., Ltd.) were used to knockdown or overexpress PFKFB2, respectively. Cells were transfected with siRNA (30 $\mathrm{nM})$ or overexpression vectors $(1 \mu \mathrm{g})$ using Lipofectamine ${ }^{\circledR} 2000$ reagent (Invitrogen; Thermo Fisher Scientific, Inc.) for $48 \mathrm{~h}$ at $37^{\circ} \mathrm{C}$. Cells were harvested $48 \mathrm{~h}$ post-transfection for use in subsequent experiments.

Reverse transcription-quantitative PCR (RT-qPCR). Transfected or untransfected A549 and H446 cells were treated with or without $50 \mu \mathrm{M}$ shikonin (Shanghai Yuanye Biotechnology Co., Ltd.) for $24 \mathrm{~h}$ at $37^{\circ} \mathrm{C}$. Total RNA was extracted from clinical specimens or cells using TRIzol ${ }^{\circledR}$ reagent (Invitrogen; Thermo Fisher Scientific, Inc.), according to the manufacturer's protocol. Total RNA was reverse transcribed into cDNA using a PrimeScript RT kit (Takara Biotechnology Co., Ltd.), according to the manufacturer's protocol. qPCR was subsequently performed on an ABI 7500 Real-Time PCR system (Applied Biosystems; Thermo Fisher Scientific, Inc.) using a SYBR ${ }^{\circledR}$ Premix Ex Taq ${ }^{\mathrm{TM}}$ kit (Takara Biotechnology Co., Ltd.). The following thermocycling conditions were used for the qPCR: Initial denaturation at $95^{\circ} \mathrm{C}$ for $3 \mathrm{~min}$; followed by 40 cycles at $95^{\circ} \mathrm{C}$ for $5 \mathrm{sec}$ and $60^{\circ} \mathrm{C}$ for $30 \mathrm{sec}$. The following primers pairs (Sangon Biotech Co., Ltd.) were used for the qPCR: PFKFB2 forward, 5'-GCTGCTTGG TGGGAGTGATAA-3' and reverse, 5'-TGAGAAGCCAAG TGTCAGGG-3'; and $\beta$-actin forward, 5'-GAGGACCCTGGA TGTGACAG-3' and reverse, 5'-AAGACCTGTACGCCAACA CA-3'. Relative mRNA expression levels were quantified using the $2^{-\Delta \Delta \mathrm{Cq}}$ method (27) and normalized to $\beta$-actin.

Western blotting. A549 and H446 cells were treated with shikonin [0 (control), 10, 20 or $50 \mu \mathrm{M}$ ] for $24 \mathrm{~h}$ at $37^{\circ} \mathrm{C}$, then total protein was extracted from cells using RIPA lysis buffer (Santa Cruz Biotechnology, Inc.) supplemented with protease and phosphatase inhibitor mixture. Total protein was quantified using a BCA assay and $50 \mu \mathrm{g}$ protein/lane was separated via $10 \%$ SDS-PAGE. The separated proteins were subsequently transferred onto PVDF membranes (EMD Millipore) and blocked with $5 \%$ skim milk for $2 \mathrm{~h}$ at room temperature. The membranes were then incubated with the following primary antibodies at $4^{\circ} \mathrm{C}$ overnight: Anti-PFKFB2 (1:1,000; cat. no. ab234865; Abcam), anti-PDK1 (1:2,000; cat. no. ab202468; Abcam), anti-glucose transporter 1 (GLUT1; 1:200; cat.no.ab150299; Abcam), anti-phosphoglycerate kinase 2 (PGK2; 1:1,000; cat. no. ab183031; Abcam), anti-lactate dehydrogenase A (LDHA; 1:1,000; cat. no. ab101562; Abcam), anti-PKM2 (1:1,000; cat. no. ab137852; Abcam), anti-GLUT3 (1:8,000; cat. no. ab41525; Abcam), anti-pyruvate dehydrogenase (PDH; 1:1,000; cat. no. 3205; Cell Signaling Technology, Inc.), phosphorylated (p)-PDH (1:1,000; cat. no. 31866; Cell Signaling Technology, Inc.) and anti-GAPDH (1:2,500; cat. no. ab9485; Abcam). Following the primary antibody incubation, the membranes were incubated with an anti-rabbit HRP-conjugated secondary antibody (1:5,000; cat. no. sc-2357; Santa Cruz Biotechnology, Inc.) for $1 \mathrm{~h}$ at room temperature. Protein bands were visualized using an enhanced chemiluminescence kit (EMD Millipore). 
Cell proliferation assay. Following transfection, A549 and H446 cells were plated into 96 -well plates at a density of $2 \times 10^{3}$ cells/well and cultured in DMEM supplemented with $10 \%$ FBS. For certain experiments, cells were also treated with shikonin $[0,10,20$ or $50 \mu \mathrm{M}]$ for $24,48,72$ or $96 \mathrm{~h}$ at $37^{\circ} \mathrm{C}$. At each time point, Cell Counting Kit-8 (CCK-8) reagent (Dojindo Molecular Technologies, Inc.) was added to each well and incubated for $2 \mathrm{~h}$ at $37^{\circ} \mathrm{C}$. The optical density (OD) value was measured at a wavelength of $450 \mathrm{~nm}$ using a microplate reader (Thermo Fisher Scientific). The experiment was repeated in triplicate.

Wound healing assay. A549 and H446 cells were digested using $0.25 \%$ trypsin solution (Gibco; Thermo Fisher Scientific, Inc.) and cultured in a 6 -well plate at a density of $5 \times 10^{5}$ cells/well overnight at $37^{\circ} \mathrm{C}$. Upon cells reaching $90 \%$ confluence, a single vertical scratch was made in the cell monolayer with a 10- $\mu 1$ micropipette tip. The cells were washed with PBS thrice to remove the detached cells. Subsequently, A549 and H446 cells were cultured in serum-free DMEM with/without shikonin $(0,10,20$ or $50 \mu \mathrm{M})$ at $37^{\circ} \mathrm{C}$ or transfected A549 cells were cultured in serum-free DMEM with $50 \mu \mathrm{M}$ shikonin at $37^{\circ} \mathrm{C}$ with $5 \% \mathrm{CO}_{2}$ in an incubator. At $0 \mathrm{~h}$ and following $24 \mathrm{~h}$ of incubation, the wound area was visualized and photographed using a light microscope (magnification, x100) and the migration was analyzed using ImageJ software (version 1.8.0; National Institutes of Health).

Colony formation assay. After transfection for 48 h, A549 and H446 cells were plated into 6-well plates at a density of $0.5-1 \times 10^{3}$ cells/well; three wells were plated for each experimental condition. The cells were cultured in complete medium supplemented with $30 \% \mathrm{FBS}$ in an incubator with $37^{\circ} \mathrm{C}$ for 14 days; the medium was changed every 3 days. During the incubation, the cell state and colony size were observed under a light microscope (magnification, x100). After culture, cells were washed with PBS, fixed with $4 \%$ paraformaldehyde for $20 \mathrm{~min}$ at room temperature and stained with $0.2 \%$ crystal violet for $5 \mathrm{~min}$ at room temperature. The cells were then washed with water, dried, photographed and counted using ImageJ software; $>50$ cells counted as a colony.

Transwell assay. Transwell assay was used to analyze cell invasion and migration. Transwell chambers (Corning, Inc.) were precoated with (invasion) or without (migration) Matrigel (Becton, Dickinson and Company) at $37^{\circ} \mathrm{C}$ for $30 \mathrm{~min}$. Following cell digestion using $0.25 \%$ trypsin solution, the culture medium was discarded by centrifugation $(300 \mathrm{x} g$ for $5 \mathrm{~min}$ at $37^{\circ} \mathrm{C}$ ) and the cell pellet was washed with PBS 1-2 times. A549 cells were treated with shikonin $(0,10,20$ or $50 \mu \mathrm{M}$ ) for $24 \mathrm{~h}$ at $37^{\circ} \mathrm{C}$. Next, cells were incubated in serum-free DMEM at a density of $5 \times 10^{5}$ cells $/ \mathrm{ml}$ for $24 \mathrm{~h}$ at $37^{\circ} \mathrm{C}$, then $100 \mu \mathrm{l}$ cell suspension was added to the upper chamber of the Transwell plate. A total of $600 \mu 1$ medium supplemented with $20 \%$ FBS was plated into the lower chambers. Following incubation for $24 \mathrm{~h}$ at $37^{\circ} \mathrm{C}$, the cells were fixed with $4 \%$ paraformaldehyde for $10 \mathrm{~min}$ at room temperature and stained with $0.1 \%$ crystal violet for $10 \mathrm{~min}$ at room temperature. Finally, the cell numbers were counted under a light microscope (magnification, x200) using ImageJ software.
Flow cytometric analysis of apoptosis. Cell apoptosis was analyzed using an Annexin V-FITC/propidium iodide (PI) staining kit (Invitrogen; Thermo Fisher Scientific, Inc.). Briefly, A549 cells were treated with shikonin $(0,10,20$ or $50 \mu \mathrm{M})$ for $24 \mathrm{~h}$ at $37^{\circ} \mathrm{C}$ or transfected A549 cells were treated with $50 \mu \mathrm{M}$ shikonin for $24 \mathrm{~h}$ at $37^{\circ} \mathrm{C}$ and collected by centrifugation $\left(300 \mathrm{x} \mathrm{g}\right.$ for $5 \mathrm{~min}$ at $\left.37^{\circ} \mathrm{C}\right)$. Cells $\left(1 \times 10^{5}\right.$ cells/well) were then washed with cold $\left(4^{\circ} \mathrm{C}\right)$ PBS twice and resuspended in $1 \mathrm{X}$ binding buffer $(200 \mu \mathrm{l})$. Cells were subsequently incubated with $5 \mu \mathrm{l}$ Annexin V-FITC/PI at room temperature in the dark for $15 \mathrm{~min}$. Apoptotic cells (early and late apoptotic cells) were visualized using a flow cytometer (Accuri ${ }^{\text {TM }}$ C6 Plus; BD Biosciences).

Measurement of lactic acid production, glucose uptake and cellular ATP levels. A549 and H446 cells (1x104 cells/well) were seeded into 6-well plates and cultured in DMEM. A549 and $\mathrm{H} 446$ cells were treated with shikonin $(0,10,20$ or $50 \mu \mathrm{M})$ for $24 \mathrm{~h}$ at $37^{\circ} \mathrm{C}$ or transfected A549 cells were treated with $50 \mu \mathrm{M}$ shikonin for $24 \mathrm{~h}$ at $37^{\circ} \mathrm{C}$, then cell culture medium was subsequently collected and centrifuged at 8,000 $\mathrm{x}$ g for $5 \mathrm{~min}$ at room temperature. The supernatant was used to measure lactate or glucose concentrations, or cellular ATP levels. Extracellular lactate levels were measured using a lactate analysis kit (cat. no. E4341; BioVision, Inc.) according to the manufacturer's protocol. Cell lysates were used to measure glucose levels using a glucose assay kit (cat. no. K686; BioVision, Inc.) according to the manufacturer's protocol. ATP levels were measured using the CellTiter-Glo ${ }^{\circledR}$ luminescent cell activity assay kit (cat. no. G7572; Promega Corporation) according to the manufacturer's protocol. The relative value of lactate, glucose uptake and ATP levels were normalized to the control group (set to 1).

Statistical analysis. Statistical analysis was performed using SPSS 21.0 software (IBM Corp.) and data are expressed as the mean \pm SEM of at least three independent experiments. Statistical differences between groups were determined using a one-way ANOVA followed by a Tukey's post hoc test. A paired Student's t-test was used to analyze the mRNA expression levels of PFKFB2 between NSCLC and adjacent normal tissues. $\mathrm{P}<0.05$ was considered to indicate a statistically significant difference.

\section{Results}

Shikonin inhibits the proliferation, invasion and migration of A549 cells. To investigate the antitumor effects of shikonin, the inhibitory effect of shikonin was determined using cellular functional experiments. The results of the cell proliferation experiment revealed that shikonin $(10,20$ or $50 \mu \mathrm{M})$ could inhibit the proliferation of A549 cells in a dose-dependent manner compared with the control group (all $\mathrm{P}<0.05$; Fig. 1A). The results from the wound healing assay demonstrated that 10,20 or $50 \mu \mathrm{M}$ shikonin could significantly inhibit the migration of A549 cells compared with the control group (all $\mathrm{P}<0.05$; Fig. 1B). The results of the Transwell assays showed that 10, 20 or $50 \mu \mathrm{M}$ shikonin could significantly inhibit the migration and invasion of A549 cells in a dose-dependent manner compared with the control group (all $\mathrm{P}<0.05$; Fig. 1C). Analysis of cell 



Figure 1. Effect of shikonin on A549 cells. (A) A549 cells were treated with 0, 10, 20 or $50 \mu \mathrm{M}$ shikonin for $24,48,72$ or $96 \mathrm{~h}$, and cell proliferation was measured using a Cell Counting Kit-8 assay. ${ }^{~} \mathrm{P}<0.05$. (B) A549 cells were treated with 0, 10, 20 or $50 \mu \mathrm{M}$ shikonin for $24 \mathrm{~h}$, and the cell migration ability was analyzed using a wound healing assay. Scale bar, 100- $\mu \mathrm{m}$. (C) A549 cells were treated with $0,10,20$ or $50 \mu \mathrm{M}$ shikonin for $24 \mathrm{~h}$, and the cell invasion and migration abilities were measured using Transwell assays. Scale bar, 100- $\mu \mathrm{m}$. (D) A549 cells were treated with 0, 10, 20 or $50 \mu \mathrm{M}$ shikonin for $24 \mathrm{~h}$, and cell apoptosis was analyzed using flow cytometry. ${ }^{*} \mathrm{P}<0.05$. OD, optical density; PI, propidium iodide.

apoptosis revealed that 10,20 or $50 \mu \mathrm{M}$ shikonin increased the apoptosis of A549 cells compared with the control group (all $\mathrm{P}<0.05$; Fig. 1D).

Shikonin inhibits the Warburg effect in a dose-dependent manner and regulates glucose metabolism in A549 and $\mathrm{H} 446 \mathrm{cells}$. To further determine the underlying mechanism of the effect of shikonin on lung cancer cells, the effect of shikonin on the Warburg effect and glucose metabolism was investigated in A549 and $\mathrm{H} 446$ cells. The results found that 10,20 or $50 \mu \mathrm{M}$ shikonin could dose-dependently reduce glucose uptake, lactate and ATP levels in A549 cells compared with the control group (all $\mathrm{P}<0.05$; Fig. 2A-C). Similar results were obtained for $\mathrm{H} 446$ cells (all $\mathrm{P}<0.05$; Fig. 2D-F). These data indicated that shikonin may inhibit the Warburg effect and regulate glucose metabolism in lung cancer cells.
PFKFB2 expression is regulated by shikonin. To determine the mechanism through which shikonin inhibits glycolysis and the migration, proliferation and invasion of tumor cells, the protein expression levels of key enzymes involved in aerobic glucose metabolism were analyzed in A549 and H446 cells treated with shikonin. As shown in Fig. 3A, western blotting analysis revealed that shikonin downregulated the protein expression levels of PFKFB2 in a dose-dependent manner in A549 and H446 cells, while the expression levels of the other proteins (PDK1, GLUT1, PGK2, LDHA, PKM2, GLUT3, PDH and p-PDH) were not altered by shikonin treatment. These findings indicated that PFKFB2 expression may be regulated by shikonin. In addition, RT-qPCR analysis was performed to analyze the mRNA expression levels of PFKFB2 in A549 and H446 cells treated with $50 \mu \mathrm{M}$ shikonin. The results revealed that treatment with shikonin significantly downregulated the mRNA 

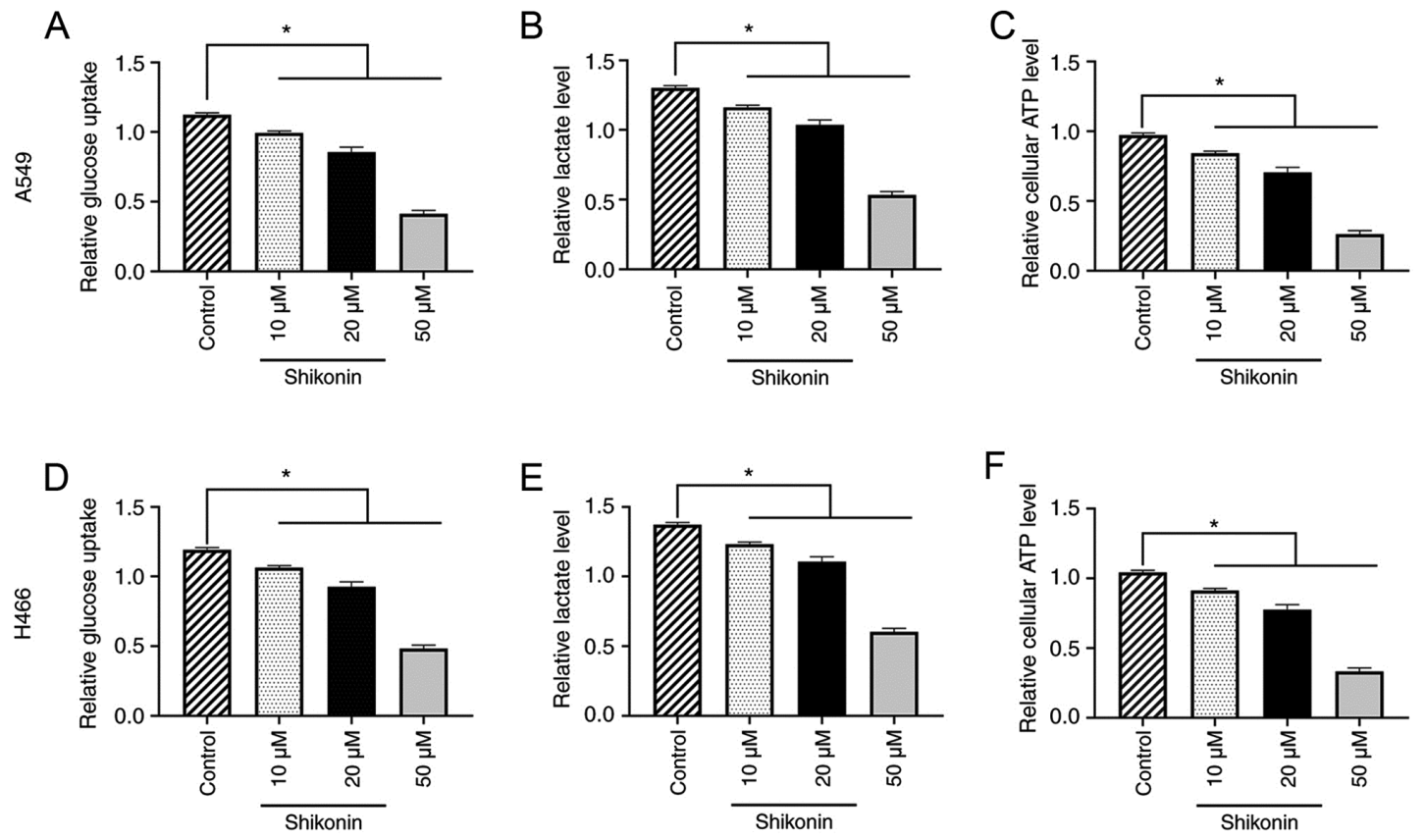

Figure 2. Shikonin inhibits the Warburg effect and regulates the glycometabolism of A549 and H446 cells in a dose-dependent manner. A549 and H446 cells were treated with $0,10,20$ or $50 \mu \mathrm{M}$ shikonin for $24 \mathrm{~h}$. Shikonin inhibited (A) glucose uptake, (B) lactate levels and (C) ATP levels in A549 cells. Shikonin inhibited (D) glucose uptake, (E) lactate levels and (F) ATP levels in H446 cells. "P<0.05.
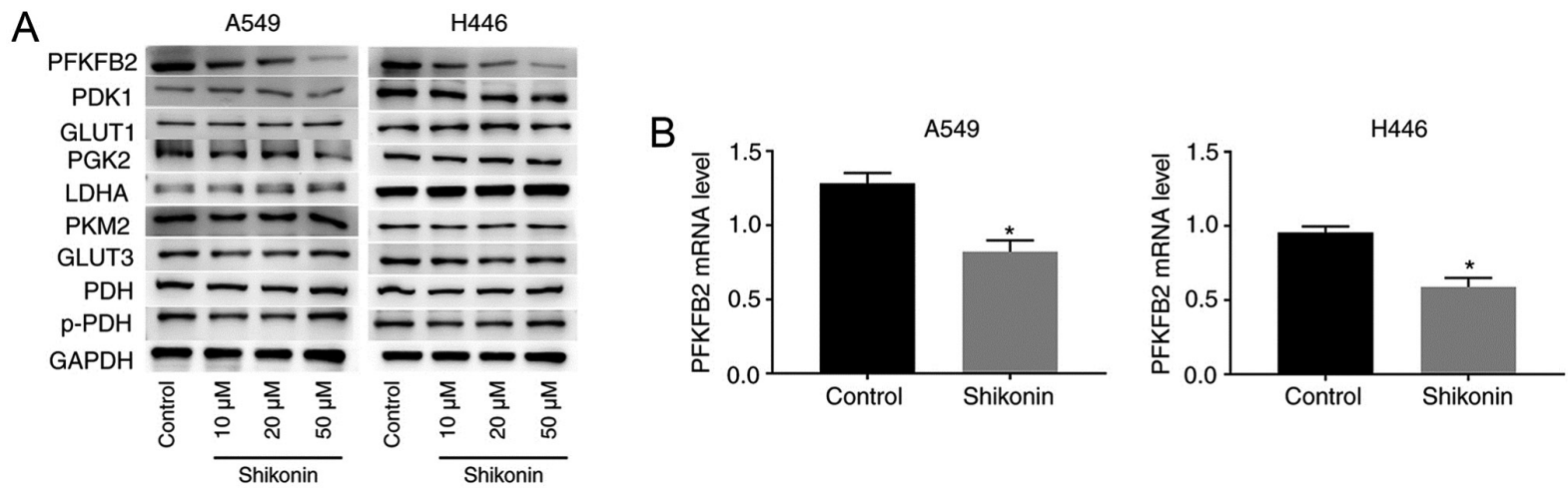

Figure 3. Shikonin downregulates PFKFB2 expression levels in A549 and H446 cells. (A) A549 and H446 cells were incubated with 0, 10, 20 or $50 \mu \mathrm{M}$ shikonin for $24 \mathrm{~h}$. Expression levels of PFKFB2, PDK1, GLUT1, PGK2, LDHA, PKM2, GLUT3, PDH and p-PDH in A549 and H446 cells were analyzed using western blotting. (B) mRNA expression levels of PFKFB2 were analyzed using reverse transcription-quantitative PCR in A549 and H446 cells treated with $50 \mu \mathrm{M}$ shikonin. "P<0.05 vs. control. PFKFB2, 6-phosphofructo-2-kinase/fructose-2,6-biphosphatase 2; PDK1, pyruvate dehydrogenase kinase 1; GLUT, glucose transporter; PGK2, phosphoglycerate kinase 2; LDHA, lactate dehydrogenase A; PKM2, pyruvate kinase M1/2; PDH, pyruvate dehydrogenase phosphatase catalytic subunit $1 ; \mathrm{p}-$, phosphorylated.

expression levels of PFKFB2 compared with the control group (both $\mathrm{P}<0.05$; Fig. 3B).

Expression levels of PFKFB2 are upregulated in lung cancer tissues, and the overexpression of PFKFB2 promotes proliferation, migration and the Warburg effect in lung cancer cells. To investigate the role of PFKFB2, the expression levels of PFKFB2 were first analyzed in 20 NSCLC and adjacent normal tissues. The results demonstrated that PFKFB2 expression levels were significantly upregulated in NSCLC tissues compared with the adjacent normal tissues $(\mathrm{P}<0.05$; Fig. 4A). The expression levels of PFKFB2 were also significantly upregulated in lung cancer cells (A549 and H446) compared with Beas-2B normal lung epithelial cells (both $\mathrm{P}<0.05$; Fig. 4B). In addition, RT-qPCR analysis found that PFKFB2 mRNA expression levels were significantly downregulated in A549 and H446 cells transfected with siRNA-PFKFB2 compared with siRNA-NC (both $\mathrm{P}<0.05$ ) and significantly upregulated in A549 and H446 cells transfected with pcDNA3.1-PFKFB2 compared with pcDNA3.1-NC (both $\mathrm{P}<0.05$ ) (Fig. 4C), which indicated that the cell transfections were successful. The results of the CCK- 8 and colony formation assays revealed that the overexpression of PFKFB2 significantly increased the proliferation of A549 and H446 cells compared with the pcDNA3.1-NC group, while the knockdown of PFKFB2 significantly inhibited the proliferation of A549 and H446 cells compared with the siRNA-NC group (all $\mathrm{P}<0.05$; Fig. 4D and E). The results of the wound healing assay demonstrated that the overexpression of PFKFB2 significantly promoted the migration of A549 and 



Figure 4. PFKFB2 expression levels are upregulated in lung cancer, and the overexpression of PFKFB2 promotes proliferation, migration and the Warburg effect in A549 and H446 cells. (A) mRNA expression levels of PFKFB2 in NSCLC and adjacent normal tissues were analyzed using RT-qPCR. (B) mRNA expression levels of PFKFB2 were analyzed in Beas-2B, A549 and H446 cells using RT-qPCR. (C) mRNA expression levels of PFKFB2 in A549 and H446 cells following transfections were analyzed using RT-qPCR. (D) Proliferation of A549 and H446 cells was detected using a Cell Counting Kit-8 assay. (E) Colony formation assay was used to measure the colony number in A549 and H446 cells. (F) Migratory ability of A549 and H446 cells was analyzed using a wound healing assay. Scale bar, 100- $\mu \mathrm{m}$. "P<0.05. PFKFB2, 6-phosphofructo-2-kinase/fructose-2,6-biphosphatase 2; RT-qPCR, reverse transcription-quantitative PCR; NSCLC, non-small cell lung cancer; siRNA, small interfering RNA; NC, negative control; OD, optical density. 

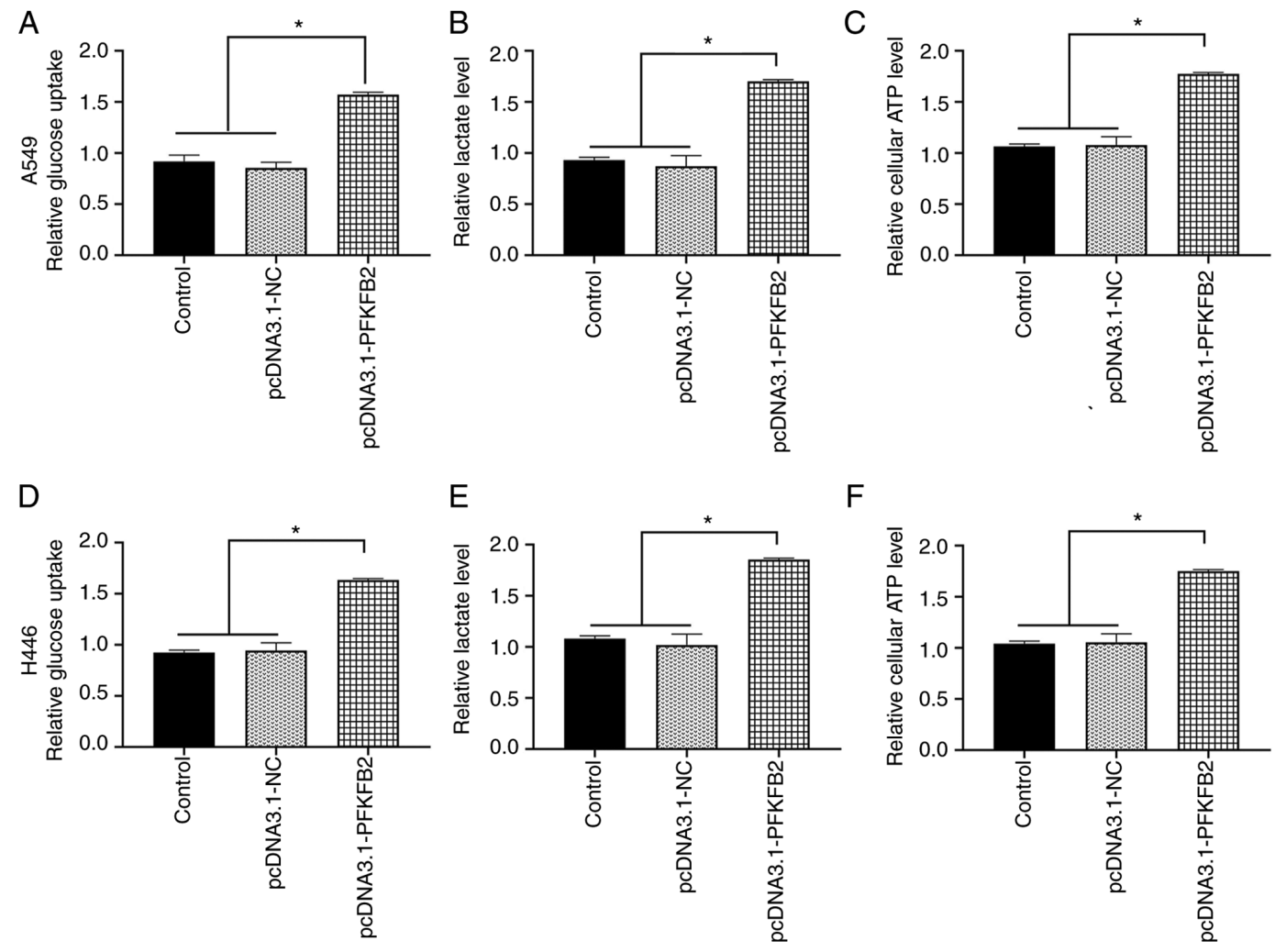

Figure 5. Overexpression of PFKFB2 accelerates the Warburg effect in lung cancer cells. (A) Overexpression of PFKFB2 in A549 cells increased (A) glucose uptake, (B) lactate levels and (C) ATP levels. Overexpression of PFKFB2 in H446 cells increased (D) glucose uptake, (E) lactate levels and (F) ATP levels. ${ }^{*} \mathrm{P}<0.05$. PFKFB2, 6-phosphofructo-2-kinase/fructose-2,6-biphosphatase 2; NC, negative control.

H446 cells compared with the pcDNA3.1-NC group, while the knockdown of PFKFB2 significantly inhibited the migration of A549 and H446 cells compared with the siRNA-NC group (all P<0.05; Fig. 4F).

Subsequently, whether PFKFB2 regulated the glycometabolic activity in A549 and $\mathrm{H} 446$ cells was investigated. The overexpression of PFKFB2 in A549 cells significantly increased glucose uptake, lactate and ATP levels compared with the control and pcDNA3.1-NC groups (all $\mathrm{P}<0.05$; Fig. 5A-C). Similar results were obtained for $\mathrm{H} 446$ cells (all $\mathrm{P}<0.05$; Fig. 5D-F).

PFKFB2 participates in shikonin-induced glycolysis, apoptosis and migration in lung cancer cells. Whether PFKFB2 participated in shikonin-induced aerobic glycolysis, cell apoptosis and migration was subsequently investigated. As shown in Fig. 6A-D, the knockdown of PFKFB2 or treatment of shikonin significantly inhibited glucose uptake, lactic acid production, ATP levels and migration in A549 cells compared with the control group (all $\mathrm{P}<0.05$ ). Notably, there were no significant differences in glucose uptake, lactate levels, ATP production and migration between A549 cells with knocked down PFKFB2 or treated with shikonin and the knockdown of PFKFB2 in A549 cells treated with shikonin, suggesting that shikonin-mediated glycolysis and migration may be regulated by PFKFB2 in lung cancer cells. In addition, the knockdown of PFKFB2 or treatment of shikonin significantly increased cell apoptosis compared with the control group $(\mathrm{P}<0.05)$, while the knockdown of PFKFB2 in shikonin-treated cells did not significantly alter the apoptosis compared with treatment of shikonin or the knockdown of PFKFB2 (Fig. 6E).

\section{Discussion}

Lung cancer has become the most commonly diagnosed cancer worldwide, accounting for $11.6 \%$ of total cancers diagnosed (1). Currently, due to the lack of effective tumor molecular markers available to guide clinical diagnosis and treatment, the rate of successful treatment in patients with lung cancer remains poor; therefore, it remains an urgent priority to develop novel therapeutic drugs (28). Traditional Chinese medicine has long been used for the treatment of lung cancer and has demonstrated considerable clinical value, such as inhibiting metastasis, enhancing the host immune response and reducing the adverse effects of chemotherapy (29-31). The results of the present study revealed that shikonin inhibited cell proliferation, invasion, and migration, reduced glucose uptake, lactate and ATP levels, increased apoptosis and downregulated the expression levels of PFKFB2 in lung cancer cells.

Shikonin, a natural naphthoquinone compound, is extracted and separated from the dry roots of Lithospermum erythrorhizon and has been found to play roles in numerous biological activities, including exerting anti-inflammatory, antiviral and antitumor effects, immune system regulation, promoting wound healing and protecting against multi-drug resistance (32-34). Among these biological activities, the anticancer effect of shikonin has been well reported. For example, a previous study reported that shikonin reduced the tumor diameter by $25 \%$ and increased the 1-year survival rate by $47.3 \%$ in patients with lung cancer (35). In addition, Zhang et al (36) demonstrated that shikonin inhibited the migration and invasion of thyroid cancer cells. Guo et al (37) also found that shikonin 

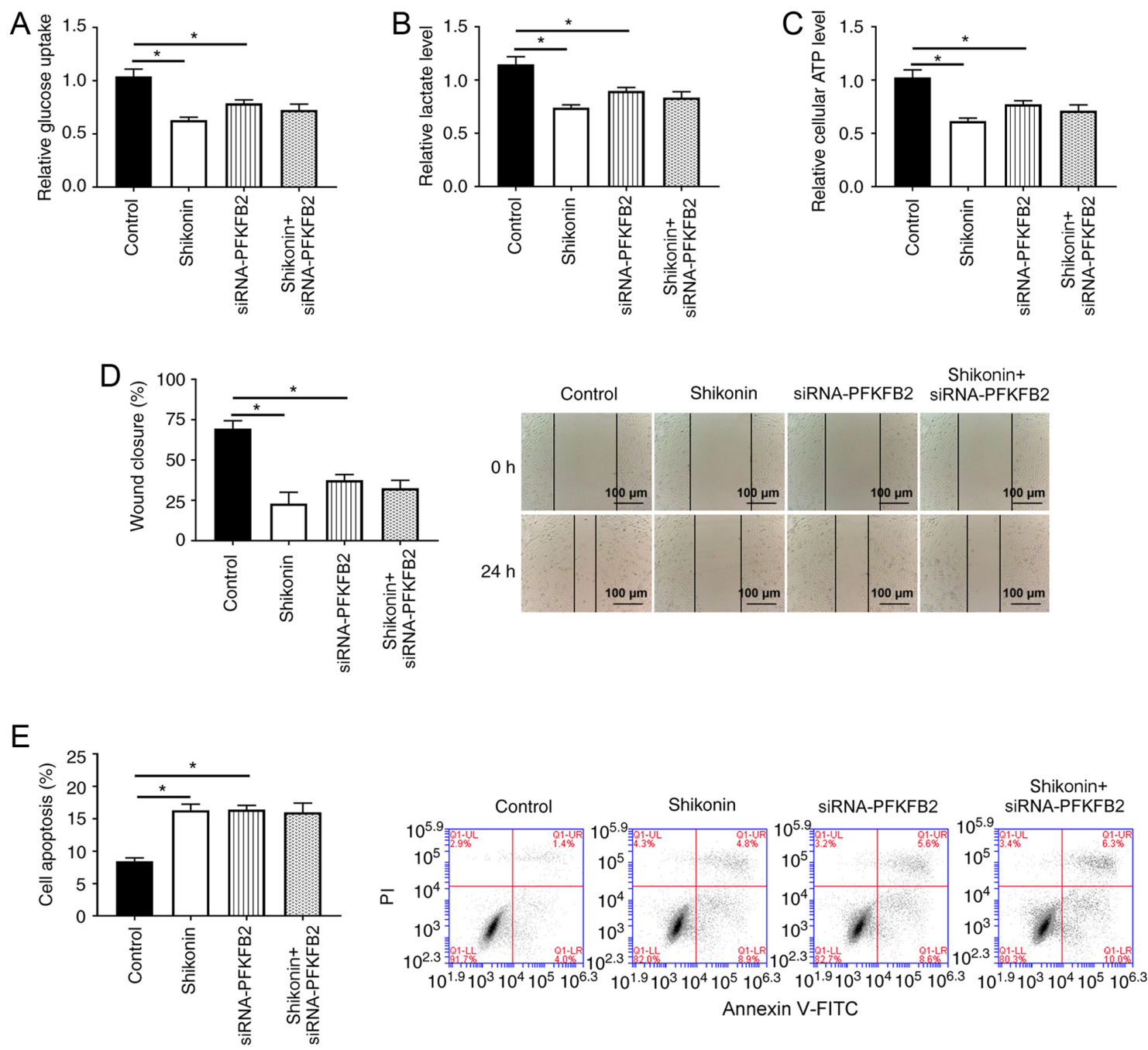

Figure 6. PFKFB2 participates in the shikonin-induced effects on glycometabolism, apoptosis and migration. A549 cells were incubated with $50 \mu \mathrm{M}$ shikonin for 24 h. (A) Glucose uptake, (B) lactate levels and (C) ATP levels were analyzed in A549 cells. (D) Cell migration ability of A549 cells was analyzed using a wound healing assay. Scale bar, 100- $\mu \mathrm{m}$. (E) Flow cytometry was used to analyze the apoptosis of A549 cells. "P<0.05. PFKFB2, 6-phosphofructo-2-kinase/fructose-2,6-biphosphatase 2; siRNA, small interfering RNA; NC, negative control; NS, non-significant; PI, propidium iodide.

inhibited cell proliferation and induced apoptosis in glioma. Wang et al (38) reported that shikonin inhibited the proliferation and promoted apoptosis of breast cancer cells. The results of the present study demonstrated that shikonin could inhibit proliferation, migration and invasion, and increase the apoptosis of A549 cells in a dose-dependent manner, which were consistent with the findings of previous studies (36-38). However, the results of the present study showed a lower apoptosis rate, which is different to the results obtained in the aforementioned studies. Taken together, these results indicated that shikonin might exert antitumor effects in lung cancer.

Cancer cells do not use mitochondrial oxidative phosphorylation, even in the presence of oxygen, but instead use aerobic glycolysis, a phenomenon termed the Warburg effect $(39,40)$. The Warburg effect has been reported to permit tumor cells to overcome metabolic stress and promote energy replenishment, which is vital for the proliferation and survival of cancer cells $(41,42)$. In addition, the Warburg effect was suggested to impact the microenvironment and promote chemoresistance, angiogenesis and metastasis $(43,44)$. Hence, investigating the Warburg effect may be crucial for determining the underlying mechanisms of the development and progression of NSCLC. The results of the present study found that shikonin could dose-dependently reduce glucose uptake, lactate and ATP levels in lung cancer cells. These data indicated that shikonin may inhibit the malignant evolution of lung cancer by inhibiting the Warburg effect.

PFKFB is an important regulator of glycolysis in cancer, and it was previously reported that the PFKFB family served an important role in the development and progression of numerous tumor types, such as lung, pancreatic and gastric cancer (45-47). Previous studies demonstrated that the expression levels of PFKFB2 were upregulated in melanoma, osteosarcoma and thyroid carcinoma (48-50). Furthermore, Liu et al (51) reported that microRNA-613 inhibited cell proliferation, invasion and the Warburg effect by regulating PFKFB2 expression in gastric cancer. Ozcan et al (22) also found that PFKFB2 inhibited glycolysis and proliferation in pancreatic cancer cells. The present study results showed that the expression levels of PFKFB2 were significantly upregulated in human NSCLC tissues and lung cancer cell lines, and that the overexpression of PFKFB2 increased cell proliferation, migration, glucose uptake, 
lactate and ATP levels in lung cancer cells. In addition, shikonin treatment downregulated the expression levels of PFKFB2. Furthermore, there were no significant differences in glucose uptake, lactate levels, ATP production, apoptosis and migration between the knockdown of PFKFB2 or treatment of shikonin and the knockdown of PFKFB2 in cells treated with shikonin. These results indicated that PFKFB2 may play an important role in the effects of shikonin treatment in lung cancer.

To the best of our knowledge, the present study was the first to demonstrate that shikonin inhibited lung cancer cell migration and the Warburg effect by regulating PFKFB2 expression, which may provide a novel insight into the mechanism underlying the anticancer effects of shikonin. However, it is important to note the limitations of the present study. For example, the proportion of apoptotic cells was too low to ascertain that shikonin induced apoptosis. In addition, the current study did not block the Warburg effect to further validate the effect of shikonin on cell proliferation, apoptosis and migration. The above limitations may weaken the conclusions of the present study; therefore, koningic acid, an irreversible and selective inhibitor of GAPDH (a rate-controlling glycolytic enzyme during the Warburg effect) will be used in future studies to block the Warburg effect to further validate the mechanism of shikonin in lung cancer (52).

In conclusion, the results of the present study suggested that shikonin inhibited the metabolism, migration and proliferation of lung cancer cells. In lung cancer tissues, PFKFB2 expression levels were upregulated and promoted the progression of lung cancer. In addition, PFKFB2 was found to participate in the shikonin-induced effects on the glycolysis and migration of lung cancer cells. These data suggested that treatments targeting PFKFB2 may benefit patients with lung cancer. In addition, shikonin may represent a potential novel compound for the treatment of lung cancer.

\section{Acknowledgements}

Not applicable.

\section{Funding}

No funding was received.

\section{Availability of data and materials}

The datasets used and/or analyzed during the current study are available from the corresponding author on reasonable request.

\section{Authors' contributions}

HZ conceived and designed the study; LS, ZL and XS performed the experiments; TW, YL and YZ analyzed and interpreted the data; and all authors wrote the manuscript. All authors read and approved the final manuscript. LS and HZ confirm the authenticity of all the raw data.

\section{Ethics approval and consent to participate}

The study protocols were approved by the Ethics Committee of The Affiliated Hospital of Qingdao University, and written informed consent was obtained from all patients prior to participation.

\section{Patient consent for publication}

Not applicable.

\section{Competing interests}

The authors declare that they have no competing interests.

\section{References}

1. Bray F, Ferlay J, Soerjomataram I, Siegel RL, Torre LA and Jemal A: Global cancer statistics 2018: GLOBOCAN estimates of incidence and mortality worldwide for 36 cancers in 185 countries. CA Cancer J Clin 68: 394-424, 2018.

2. Blandin Knight S, Crosbie PA, Balata H, Chudziak J, Hussell T and Dive C: Progress and prospects of early detection in lung cancer. Open Biol 7: 170070, 2017.

3. Rossi A, Tay R, Chiramel J, Prelaj A and Califano R: Current and future therapeutic approaches for the treatment of small cell lung cancer. Expert Rev Anticancer Ther 18: 473-486, 2018.

4. King-Kallimanis BL, Kanapuru B, Blumenthal GM, Theoret MR and Kluetz PG: Age-related differences in patient-reported outcomes in patients with advanced lung cancer receiving anti-PD-1/PD-L1 therapy. Semin Oncol 45: 201-209, 2018.

5. Shukuya T and Carbone DP: Predictive markers for the efficacy of anti-PD-1/PD-L1 antibodies in lung cancer. J Thorac Oncol 11: 976-988, 2016.

6. Festino L, Botti G, Lorigan P, Masucci GV, Hipp JD, Horak CE, Melero I and Ascierto PA: Cancer treatment with Anti-PD-1/PD-L1 agents: Is PD-L1 expression a biomarker for patient selection? Drugs 76: 925-945, 2016.

7. Zhong W, Chen S, Qin Y, Zhang H, Wang H, Meng J, Huai L, Zhang Q, Yin T, Lei Y, et al: Doxycycline inhibits breast cancer EMT and metastasis through PAR-1/NF- $\mathrm{BB} / \mathrm{miR}-17 / \mathrm{E}-\mathrm{cadh}$ rin pathway. Oncotarget 8: 104855-104866, 2017.

8. Zhong W, Chen S, Zhang Q, Xiao T, Qin Y, Gu J, Sun B, Liu Y, Jing X, Hu X, et al: Doxycycline directly targets PAR1 to suppress tumor progression. Oncotarget 8: 16829-16842, 2017.

9. Liberti MV and Locasale JW: The Warburg effect: How does it benefit cancer cells? Trends Biochem Sci 41: 211-218, 2016.

10. Bi YH, Han WQ, Li RF, Wang YJ, Du ZS, Wang XJ and Jiang Y: Signal transducer and activator of transcription 3 promotes the Warburg effect possibly by inducing pyruvate kinase M2 phosphorylation in liver precancerous lesions. World J Gastroenterol 25: 1936-1949, 2019.

11. Luo Y, Medina Bengtsson L, Wang X, Huang T, Liu G, Murphy S, Wang C, Koren J III, Schafer Z and Lu X: UQCRH downregulation promotes Warburg effect in renal cell carcinoma cells. Sci Rep 10: 15021, 2020.

12. Martinez-Outschoorn UE, Lin Z, Trimmer C, Flomenberg N, Wang C, Pavlides S, Pestell RG, Howell A, Sotgia F and Lisanti MP: Cancer cells metabolically 'fertilize' the tumor microenvironment with hydrogen peroxide, driving the Warburg effect: Implications for PET imaging of human tumors. Cell Cycle 10: 2504-2520, 2011.

13. Gregory CD and Paterson M: An apoptosis-driven 'onco-regenerative niche': Roles of tumour-associated macrophages and extracellular vesicles. Philos Trans R Soc Lond B Biol Sci 373: 20170003, 2018.

14. Chen Z, Zuo X, Yao Z, Han G, Zhang L, Wu J and Wang X: MiR-3662 suppresses hepatocellular carcinoma growth through inhibition of HIF-1 $\alpha$-mediated Warburg effect. Cell Death Dis 9: 549, 2018

15. Wang S, Zhang Y, Cai Q, Ma M, Jin LY, Weng M, Zhou D, Tang Z, Wang JD and Quan Z: Circular RNA FOXP1 promotes tumor progression and Warburg effect in gallbladder cancer by regulating PKLR expression. Mol Cancer 18: 145, 2019.

16. Li J, Zhang J, Xie F, Peng J and Wu X: Macrophage migration inhibitory factor promotes Warburg effect via activation of the NF- $\kappa \mathrm{B} / \mathrm{HIF}-1 \alpha$ pathway in lung cancer. Int J Mol Med 41: 1062-1068, 2018.

17. Lu J, Tan M and Cai Q: The Warburg effect in tumor progression: Mitochondrial oxidative metabolism as an anti-metastasis mechanism. Cancer Lett 356: 156-164, 2015. 
18. Feng Y, Liu J, Guo W, Guan Y, Xu H, Guo Q, Song X, Yi F, Liu T, Zhang W, et al: Atg7 inhibits Warburg effect by suppressing PKM2 phosphorylation resulting reduced epithelial-mesenchymal transition. Int J Biol Sci 14: 775-783, 2018.

19. Xu L, Li Y, Zhou L, Dorfman RG, Liu L, Cai R, Jiang C, Tang D, Wang Y, Zou X, et al: SIRT3 elicited an anti-Warburg effect through HIF1 $\alpha /$ PDK1/PDHA1 to inhibit cholangiocarcinoma tumorigenesis. Cancer Med 8: 2380-2391, 2019.

20. Zhu W, Huang Y, Pan Q, Xiang P, Xie N and Yu H: MicroRNA-98 suppress Warburg effect by targeting HK2 in colon cancer cells Dig Dis Sci 62: 660-668, 2017.

21. Khan MA, Zubair H, Anand S, Srivastava SK, Singh S and Singh AP: Dysregulation of metabolic enzymes in tumor and stromal cells: Role in oncogenesis and therapeutic opportunities. Cancer Lett 473: 176-185, 2020.

22. Ozcan SC, Sarioglu A, Altunok TH, Akkoc A, Guzel S, Guler S, Imbert-Fernandez Y, Muchut RJ, Iglesias AA, Gurpinar Y, et al: PFKFB2 regulates glycolysis and proliferation in pancreatic cancer cells. Mol Cell Biochem 470: 115-129, 2020.

23. Yang H, Shu Z, Jiang Y, Mao W, Pang L, Redwood A, Jeter-Jones SL, Jennings NB, Ornelas A, Zhou J, et al: 6-Phosphofructo-2-kinase/fructose-2,6-biphosphatase-2 regulates TP53-dependent paclitaxel sensitivity in ovarian and breast cancers. Clin Cancer Res 25: 5702-5716, 2019.

24. Pape MR, Prokop C and Hedrich HJ: Localization of the Pfkfb 2 gene on rat Chromosome 13. Mamm Genome 7: 559, 1996.

25. Novellasdemunt L, Tato I, Navarro-Sabate A, Ruiz-Meana M, Méndez-Lucas A, Perales JC, Garcia-Dorado D, Ventura F, Bartrons R and Rosa JL: Akt-dependent activation of the heart 6-phosphofructo-2-kinase/fructose-2,6-bisphosphatase (PFKFB2) isoenzyme by amino acids. J Biol Chem 288: 10640-10651, 2013.

26. Jeung YJ, Kim HG, Ahn J, Lee HJ, Lee SB, Won M, Jung CR, Im JY, Kim BK, Park SK, et al: Shikonin induces apoptosis of lung cancer cells via activation of FOXO3a/EGR1/SIRT1 signaling antagonized by p300. Biochim Biophys Acta 1863: 2584-2593, 2016.

27. Livak KJ and Schmittgen TD: Analysis of relative gene expression data using real-time quantitative PCR and the 2(-Delta Delta C(T)) method. Methods 25: 402-408, 2001

28. Barta JA, Powell CA and Wisnivesky JP: Global epidemiology of lung cancer. Ann Glob Health 85: 8, 2019.

29. Li L, Wang S, Yang X, Long S, Xiao S, Wu W and Hann SS Traditional Chinese medicine, Fuzheng Kang-Ai decoction, inhibits metastasis of lung cancer cells through the STAT3/MMP9 pathway. Mol Med Rep 16: 2461-2468, 2017.

30. Liao YH, Li CI, Lin CC, Lin JG, Chiang JH and Li TC: Traditional Chinese medicine as adjunctive therapy improves the long-term survival of lung cancer patients. J Cancer Res Clin Oncol 143: 2425-2435, 2017.

31. Yang J, Zhu X, Yuan P, Liu J, Wang B and Wang G: Efficacy of traditional Chinese medicine combined with chemotherapy in patients with non-small cell lung cancer (NSCLC): A meta-analysis of randomized clinical trials. Support Care Cancer 28: 3571-3579, 2020

32. Andújar I, Ríos JL, Giner RM and Recio MC: Pharmacological properties of shikonin-a review of literature since 2002. Planta Med 79: 1685-1697, 2013.

33. Wang F, Yao X, Zhang Y and Tang J: Synthesis, biological function and evaluation of shikonin in cancer therapy. Fitoterapia 134 329-339, 2019

34. Wei PL, Tu CC, Chen CH, Ho YS, Wu CT, Su HY, Chen WY, Liu JJ and Chang YJ: Shikonin suppresses the migratory ability of hepatocellular carcinoma cells. J Agric Food Chem 61: 8191-8197, 2013

35. Guo XP, Zhang XY and Zhang SD: Clinical trial on the effects of shikonin mixture on later stage lung cancer. Zhong Xi Yi Jie He Za Zhi 11: 598-599, 580, 1991 (In Chinese).

36. Zhang Y, Sun B, Huang Z, Zhao DW and Zeng Q: Shikonin inhibites migration and invasion of thyroid cancer cells by downregulating DNMT1. Med Sci Monit 24: 661-670, 2018.
37. Guo N, Miao R, Gao X, Huang D, Hu Z, Ji N, Nan Y, Jiang F and Gou X: Shikonin inhibits proliferation and induces apoptosis in glioma cells via downregulation of CD147. Mol Med Rep 19: 4335-4343, 2019.

38. Wang W, Wu Y, Chen S, Liu X, He J, Wang S, Lu W, Tang Y and Huang J: Shikonin is a novel and selective IMPDH2 inhibitor that target triple-negative breast cancer. Phytother Res 35: 463-476, 2021.

39. Yu Z, Huang L, Qiao P, Jiang A, Wang L, Yang T, Tang S, Zhang W and Ren C: PKM2 Thr454 phosphorylation increases its nuclear translocation and promotes xenograft tumor growth in A549 human lung cancer cells. Biochem Biophys Res Commun 473: 953-958, 2016

40. Rong F, Tian X, Fang Z, Sun Y, Li F, Gao Y, Feng Y, Li L, Wang Y, Liu X, et al: MicroRNA-143 (miR-143) regulates cancer glycolysis via targeting hexokinase 2 gene. J Biol Chem 287: 23227-23235, 2012.

41. DeBerardinis RJ, Lum JJ, Hatzivassiliou G and Thompson CB The biology of cancer: Metabolic reprogramming fuels cell growth and proliferation. Cell Metab 7: 11-20, 2008.

42. Schild T, Low V, Blenis J and Gomes AP: Unique metabolic adaptations dictate distal organ-specific metastatic colonization. Cancer Cell 33: 347-354, 2018

43. Shamsi M, Saghafian M, Dejam M and Sanati-Nezhad A: Mathematical modeling of the function of Warburg effect in tumor microenvironment. Sci Rep 8: 8903, 2018.

44. Hussain A, Qazi AK, Mupparapu N, Guru SK, Kumar A, Sharma PR, Singh SK, Singh P, Dar MJ, Bharate SB, et al: Modulation of glycolysis and lipogenesis by novel PI3K selective molecule represses tumor angiogenesis and decreases colorectal cancer growth. Cancer Lett 374: 250-260, 2016.

45. Yalcin A, Telang S, Clem B and Chesney J: Regulation of glucose metabolism by 6-phosphofructo-2-kinase/fructose-2,6-bisphosphatases in cancer. Exp Mol Pathol 86: 174-179, 2009.

46. Minchenko OH, Ogura T, Opentanova IL, Minchenko DO, Ochiai A, Caro J, Komisarenko SV and Esumi $\mathrm{H}$ : 6-Phosphofructo-2-kinase/fructose-2,6-bisphosphatase gene family overexpression in human lung tumor. Ukr Biokhim $\mathrm{Zh} 77$ : 46-50, 1999.

47. Minchenko OH, Tsuchihara K, Minchenko DO, Bikfalvi A and Esumi H: Mechanisms of regulation of PFKFB expression in pancreatic and gastric cancer cells. World J Gastroenterol 20: 13705-13717, 2014

48. Houles T, Gravel SP, Lavoie G, Shin S, Savall M, Méant A, Grondin B, Gaboury L, Yoon SO, St-Pierre J and Roux PP: RSK regulates PFK-2 activity to promote metabolic rewiring in melanoma. Cancer Res 78: 2191-2204, 2018.

49. Zhao SJ, Shen YF, Li Q, He YJ, Zhang YK, Hu LP, Jiang YQ, Xu NW, Wang YJ, Li J, et al: SLIT2/ROBO1 axis contributes to the Warburg effect in osteosarcoma through activation of SRC/ERK/c-MYC/PFKFB2 pathway. Cell Death Dis 9: 390, 2018.

50. Camargo Barros-Filho M, Barreto Menezes de Lima L, Bisarro Dos Reis M, Bette Homem de Mello J, Moraes Beltrami C, Lopes Pinto CA, Kowalski LP and Rogatto SR: PFKFB2 promoter hypomethylation as recurrence predictive marker in well-differentiated thyroid carcinomas. Int J Mol Sci 20: 1334, 2019.

51. Liu H, Chen K, Wang L, Zeng X, Huang Z, Li M, Dong P and Chen X: miR-613 inhibits Warburg effect in gastric cancer by targeting PFKFB2. Biochem Biophys Res Commun 515: 37-43, 2019.

52. Liberti MV, Allen AE, Ramesh V, Dai Z, Singleton KR, Guo Z, Liu JO, Wood KC and Locasale JW: Evolved resistance to partial GAPDH inhibition results in loss of the Warburg effect and in a different state of glycolysis. J Biol Chem 295: 111-124, 2020.

This work is licensed under a Creative Commons Attribution-NonCommercial-NoDerivatives 4.0 International (CC BY-NC-ND 4.0) License. 